\title{
Análise comparativa de um protocolo de cicatrização secundária modificado para amputações das pontas dos dedos e técnicas de reconstrução não microcirúrgicas*
}

\section{Comparative Analysis of a Modified Secondary Healing Protocol for Fingertip Amputations and Non- microsurgical Reconstruction Techniques}

\author{
Carlos Rubén Chavez-Galvan ${ }^{10}$ Ricardo Martínez-Pérez ${ }^{10}$ Efrén Flores-Alvarez ${ }^{2}$ (1) \\ Armando Martínez-Pérez ${ }^{3}$ (i) \\ ${ }^{1}$ Departamento de Cirurgia Ortopédica e Traumatologia, Centenario \\ Endereço para correspondência Ricardo Martínez-Pérez, Cirurgião \\ Hospital Miguel Hidalgo, Aguascalientes, Aguascalientes, México \\ Ortopédico e de Trauma, Av. Ferrocarril s/n, Alameda, 20259, \\ 2 Departamento de Cirurgia Geral, Centenario Hospital Miguel \\ Hidalgo, Aguascalientes, México \\ Aguascalientes, Aguascalientes, México \\ (e-mail: ortho.surgery.martinez@gmail.com).
}

${ }^{3}$ Residente, Departamento de Ensino, Instituto Mexicano del Seguro

Social, Universidad de Guadalajara, Puerto Vallarta, Jalisco, Mexico

Rev Bras Ortop 2022;57(1):108-112.

\begin{abstract}
Resumo
Palavras-chave

- traumatismos dos dedos

- amputação

- traumatismos da mão

- cicatrização da ferida

Objetivo Descrever e comparar os resultados obtidos com um protocolo de cicatrização secundária para amputações das pontas dos dedos e sua relação com a gravidade da lesão de acordo com a classificação de Allen.

Métodos Foram revisados os prontuários clínicos de 127 lesões nas pontas dos dedos, e realizou-se um estudo retrospectivo, comparativo e analítico das amputações tratadas de forma conservadora. Foram descritas e analisadas as características da lesão, o tempo de cicatrização, e as complicações.

Resultados Entre abril de 2017 e maio de 2019, foram tratadas de forma conservadora 127 lesões nas pontas dos dedos. A idade média da amostra era de 28,33 anos. $\mathrm{O}$ tempo médio de cicatrização foi de 4,31 semanas. As complicações apresentadas durante o acompanhamento afetaram 18,9\% $(n=24)$ dos casos, porém nenhum exigiu tratamento de revisão. Não foi encontrada relação estatisticamente significativa entre o desenvolvimento das complicações e a revisão do tratamento de acordo com a classificação de Allen $(p \geq 0,05)$.
\end{abstract}

\footnotetext{
Trabalho desenvolvido no Hospital Centenario Miguel Hidalgo,

Benemérita Universidad Autónoma de Aguascalientes, México.
}

recebido

16 de Maio de 2020

aceito

18 de Maio de 2021

Publicado on-line

Outubro 25, 2021
DOI https://doi.org/ $10.1055 / \mathrm{s}-0041-1735944$. ISSN 0102-3616.
(C) 2021. Sociedade Brasileira de Ortopedia e Traumatologia. All rights reserved.

This is an open access article published by Thieme under the terms of the Creative Commons Attribution-NonDerivative-NonCommercial-License, permitting copying and reproduction so long as the original work is given appropriate credit. Contents may not be used for commercial purposes, or adapted, remixed, transformed or built upon. (https://creativecommons.org/ licenses/by-nc-nd/4.0/)

Thieme Revinter Publicações Ltda., Rua do Matoso 170, Rio de Janeiro, RJ, CEP 20270-135, Brazil 
Conclusão $\mathrm{O}$ protocolo de cicatrização secundária proposto mostrou-se seguro e eficaz nas amputações das pontas dos dedos conforme os tipos de 1 a 3 da classificação de Allen, e deve ser incluída como opção terapêutica mesmo em lesões de maior extensão do que aquelas tradicionalmente limitadas.

Abstract

\section{Keywords}

- finger injuries

- amputation

- hand injuries

- wound healing
Objective To describe and compare the results obtained with a secondary healing protocol for fingertip amputations and their relationship to injury severity according to the Allen classification.

Methods Medical records of 127 fingertip injuries were revised, and a retrospective, comparative, analytical study the amputations treated conservatively was performed. Injury characteristics, healing time, and complications were described and analyzed.

Results Between April 2017 and May 2019, 127 fingertip injuries were treated conservatively. The average age of the sample was of 28.33 years. The average healing time was of 4.31 weeks. The complications during the follow-up were observed in $18.9 \%(n=24)$ of the cases, but none require revision treatment. A statistically significant relationship between the development of complications and treatment revision according to the Allen classification was not found $(p \geq 0.05)$.

Conclusion The proposed secondary healing protocol has shown to be safe and effective in types 1 to 3 fingertip amputations in the Allen classification, and it should be included as a therapeutic option even in injuries of greater extension than those that have traditionally been limited to.

\section{Introdução}

As lesões nas pontas dos dedos das mãos estão entre as mais comuns, e resultam em aproximadamente 4,8 milhões de comparecimentos anuais aos hospitais. ${ }^{1}$ A variedade de diferentes técnicas cirúrgicas descritas para reparar pode gerar confusão, e a evolução clínica e o resultado final são afetados pelo método escolhido. As necessidades do paciente e a experiência do cirurgião devem ser levadas em consideração na tomada de decisão. ${ }^{2}$ Algumas considerações objetivas para a escolha da técnica adequada são: o ângulo de perda do tecido, a exposição óssea, a condição da parte amputada, as lesões nos dedos adjacentes, o dedo acometido, e a extensão do defeito. Embora não exista um algoritmo de tratamento preciso, os objetivos devem ser a redução da dor, a otimização do tempo de cicatrização, a preservação da sensibilidade e da função, a limitação das deformidades na lâmina ungueal, a redução do tempo de volta à atividade laboral, e, se possível, o favorecimento dos resultados estéticos, destacando-se a preservação do maior comprimento possível. Embora existam diferentes técnicas cirúrgicas para a reconstrução dos defeitos, o tratamento conservador por cicatrização secundária da ferida apresenta resultados

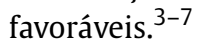

Em nosso centro hospitalar, não havia experiência relacionada ao tratamento conservador. Anteriormente, as amputações das pontas dos dedos eram tratadas com técnicas cirúrgicas convencionais (retalhos tipo V-Y, ou enxerto livre de pele), até que as técnicas conservadoras se tornassem o padrão para essas lesões, como parte de um tratamento inicial e, na maioria das vezes, como um tratamento definitivo. O objetivo deste estudo é analisar a evolução, os resultados finais e a incidência de complicações em pacientes tratados por cicatrização secundária, e comparar os resultados alcançados com os quatro tipos diferentes de lesões, segundo a classificação de Allen.

\section{Métodos}

Este estudo foi aprovado pelos comitês de ética e pesquisa da instituição. Este é um estudo observacional, retrospectivo, comparativo e analítico, realizado em pacientes com amputações traumáticas das pontas dos dedos entre abril de 2017 e maio de 2019. As pontas amputadas foram revisadas e classificadas em quatro grupos, de acordo com a classificação de Allen. O tratamento inicial foi realizado no prontosocorro, mediante técnicas assépticas e anestesia local. Todos os pacientes incluídos tiveram um período de acompanhamento de pelo menos 6 meses. Foram excluídos os pacientes que foram feridos por mordidas de animais ou seres humanos, aqueles cujo tratamento inicial foi realizado em outra instituição, e aqueles com acompanhamento incompleto. A classificação de Allen ${ }^{8}$ foi utilizada para definir a extensão das lesões. Foram descritas as características gerais da população do estudo e a duração do tratamento. As complicações precoces e tardias em cada grupo de tratamento também foram descritas, bem como foram avaliadas a necessidade de tratamento de revisão e a preservação do comprimento inicial. Foram analisadas as relações entre essas variáveis quanto ao mecanismo e as extensões das lesões, segundo a classificação de Allen e o grupo de tratamento. 


\section{Análise Estatística}

As informações resultantes foram registradas no programa Statistical Package for the Social Sciences (IBM SPSS Statistics for Windows, IBM Corp., Armonk, NY, Estados Unidos), versão 25.0. Para fins de análise estatística, foram usados os testes do Qui-quadrado e de Kruskal-Wallis. O nível de significância estabelecido foi valores de $p \leq 0,05$.

\section{Protocolo de Cicatrização Secundária}

No pronto-socorro, o dedo afetado é submetido a anestesia regional pela técnica convencional com lidocaína simples a $2 \%$. A ferida é irrigada com soro fidiológico estéril e suavemente desbridada com gaze estéril. As amputações com exposição da falange distal foram remodeladas com cinzel e lima no nível da borda da polpa. Aplicou-se o gel de ketanserina (Sufrexal, Janssen-Cilag, Beerse, Bélgica) em toda a extensão da amputação, e, em seguida, uma membrana adesiva semipermeável (Tegaderm, 3M, Saint Paiul, MN, Estados Unidos) para cobrir o defeito. Por fim, toda a circunferência do dedo, da articulação interfalangeana distal até a metacarpofalangeana, foi coberta com gaze estéril fixada com fita médica (Micropore, 3M), e uma rede elástica tubular foi fixada na base do dedo acometido. Todos os pacientes receberam antibióticos orais de forma profilática e vacinação de reforço preventiva contra o tétano. Os pacientes foram avaliados após 3 a 5 dias em ambulatório, onde realizaram a limpeza da ferida por irrigação com soro fisiológico estéril sem desbridamento; em seguida, reaplicou-se o gel de ketanserina e recobriu-se a ferida com nova membrana adesiva semipermeável, procedimento repetido semanalmente até que fosse alcançada a epitelização do defeito.

\section{Resultados}

Entre abril de 2017 e maio de 2019, 127 lesões das pontas dos dedos foram tratadas no pronto-socorro pelo serviço de trauma (-Tabela 1). Os pacientes tinham idade média de 28,33 anos (variação: 1 a 76 anos), com predominância do sexo masculino $(82,7 \% ; n=105)$. O dedo mais comumente afetado foi o dedo médio ( $31,5 \% ; n=40)$, e o mecanismo de lesão mais comum foi por esmagamento (70,9\%; $n=90)$. A maioria das lesões foi classificada como Allen tipo 2 (42,5\%; $n=54$ ) (-Fig. 1). A duração média geral do tratamento foi de 4,3 semanas, e demonstrou-se que havia uma relação estatisticamente singnificativa em proporção com a gravidade da lesão de acordo com a classificação de Allen $(p \leq 0,001)$. Do total de pontas dos dedos tratadas, $18,9 \%(n=24)$ apresentaram complicações. As 3 complicações mais frequentes foram hiperalgesia $(10,2 \% ; n=13)$, exposição persistente da falange ( $5,5 \% ; n=7)$, e seroma $(2,4 \% ; n=3)$. Apenas um paciente apresentou infecção da ferida, que foi sendo resolvida com antibioticoterapia oral de curta duração (-Fig. 2). Essas complicações apresentaram uma maior incidência nos pacientes classificados como Allen tipos 2 (45,8\%; $n=11)$, seguido pelos tipos 1 ( $25 \%$; $n=6), 4(16,6 \% ; n=4)$ e $3(12,5 \%$; $n=3)$. O número de pacientes classificados como Allen tipo 2 pode explicar a maior incidência de complicações encontra-
Tabela 1 Características da população e resultados

\begin{tabular}{|c|c|}
\hline Idade média (anos) & 28,33 \\
\hline \multicolumn{2}{|l|}{ Sexo: \% (n) } \\
\hline Masculino & $82,7(105)$ \\
\hline Feminino & $17,3(22)$ \\
\hline Diabetes mellitus: \% ( $n)$ & $0,8(1)$ \\
\hline Tabagismo: \% (n) & $12,6(12)$ \\
\hline \multicolumn{2}{|l|}{ Mecanismo da lesão: \% (n) } \\
\hline Esmagamento & $70,9(90)$ \\
\hline Cortes & $29,1(37)$ \\
\hline \multicolumn{2}{|l|}{ Classificação de Allen: \% (n) } \\
\hline Tipo 1 & $32,3(41)$ \\
\hline Tipo 2 & $42,5(54)$ \\
\hline Tipo 3 & $17,3(22)$ \\
\hline Tipo 4 & $7,9(10)$ \\
\hline \multicolumn{2}{|l|}{ Complicações: \% (n) } \\
\hline Infecção da ferida & $0,8(1)$ \\
\hline Seroma & $2,4(3)$ \\
\hline Exposição óssea persistente & $5,5(7)$ \\
\hline Ponta do dedo dolorida & $10,2(13)$ \\
\hline Necrose da ponta do dedo & - \\
\hline Dor relacionada à deformidade ungueal & - \\
\hline Revisão de tratamento: \% (n) & $6,3(8)$ \\
\hline $\begin{array}{l}\text { Encurtamento do comprimento } \\
\text { após a falha: \% (n) }\end{array}$ & $1,6(2)$ \\
\hline Tempo médio de cicatrização (semanas) & 4,31 \\
\hline
\end{tabular}

das em comparação com os pacientes Allen tipo 3. Identificou-se uma relação estatisticamente significativa $(p \leq 0,05)$ entre a classificação de Allen e o desenvolvimento de complicações (-Tabela 2).

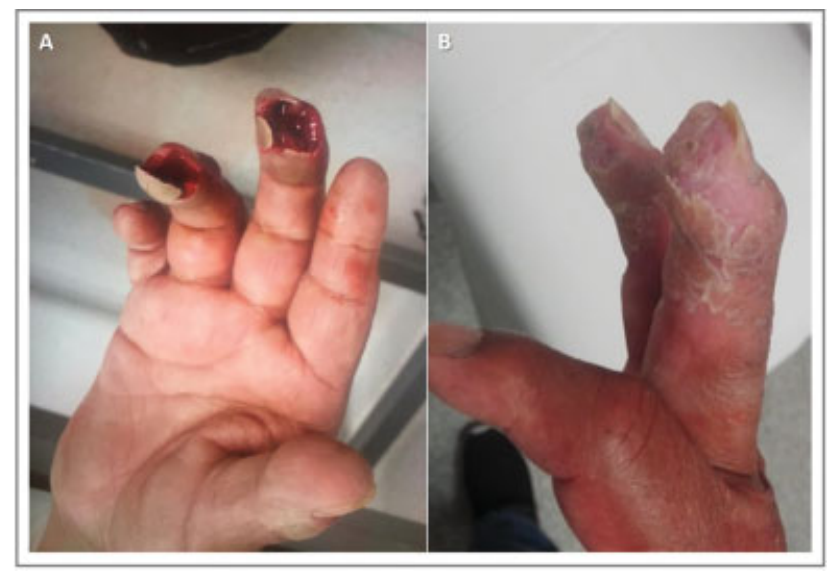

Fig. 1 Paciente do sexo masculino, de 68 anos, com lesão Allen tipo 2 , com comprometimento do terceiro e quarto dedos. (A) Apresentação aguda. (B) Após cinco semanas, mediante um protocolo de cicatrização secundária. 


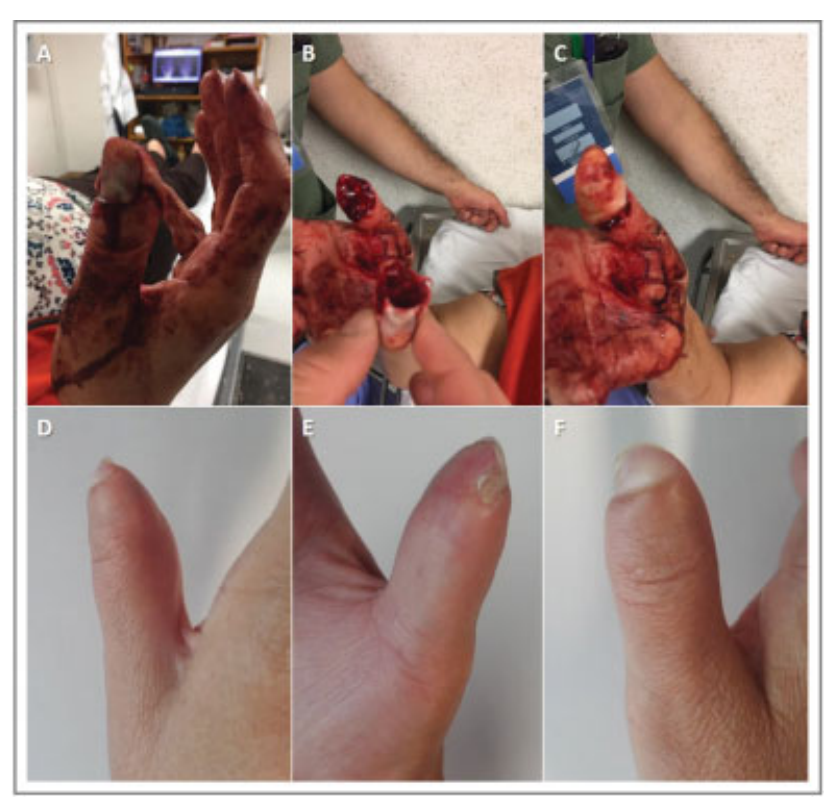

Fig. 2 Paciente do sexo feminino, de 49 anos, com lesão Allen tipo III no polegar. (A-C) Apresentação aguda. (D-F) Após seis semanas, mediante um protocolo de cicatrização secundária. Mostra-se uma deformidade ungueal assintomática.

Por fim, encontrou-se uma relação estatisticamente significativa ( $p \leq 0,001)$ entre a classificação de Allen e a preservação do comprimento máximo da ponta do dedo; a necessidade de encurtar o comprimento inicial a fim de conseguir o fechamento somente foi relatada em 2 pacientes $(1,6 \%)$ classificados como Allen tipo 2.

\section{Discussão}

As amputações das pontas dos dedos estão entre os acidentes de trabalho mais comumente tratados em todo o mundo, embora não exista um consenso quanto ao tratamento. Nos países desenvolvidos, o tratamento é cada vez mais favorável às técnicas de microcirurgia; porém, nos Estados Unidos, apenas $15 \%$ dos hospitais realizam cirurgias de reimplante. São relatadas menos de 10 cirurgias por ano, ou seja, apenas $14 \%$ das lesões nas pontas dos dedos são tratadas com esse método, ao passo que, no Japão, 29\% dos casos são tratados microcirurgicamente. ${ }^{9}$ Outra alternativa é a amputação ou a remodelação. Na série revisada neste estudo, apenas 22,7\% $(n=45)$ dos pacientes foram submetidos a remodelação como tratamento primário, ou seja, encurtamento cirúrgico para a obtenção da cicatrização secundária. Já os 77,3\% $(n=155)$ restantes foram submetidos a algum tipo de tratamento reconstrutivo conservador ou cirúrgico, que, portanto, permitiu preservar o maior comprimento possível, consequentemente obtendo-se um melhor resultado funcional e estético nos pacientes sem restrições clínicas. Esse encurtamento cirúrgico ainda é o tratamento inicial em muitos hospitais, pois permite o retorno rápido ao trabalho. Suas indicações e técnica mostram a heterogeneidade observada no tratamento das lesões nas mãos. Em um estudo ${ }^{10}$ realizado com 592 membros da American Society for Surgery of the Hand ("Sociedade Americana de Cirurgia da Mão"),
Tabela 2 Diferentes fatores e sua relação no desenvolvimento de qualquer complicação

\begin{tabular}{|c|c|c|c|}
\hline & \multicolumn{2}{|c|}{$\begin{array}{l}\text { Complicações } \\
\text { (n) }\end{array}$} & \multirow[t]{2}{*}{ Valor de $p$} \\
\hline & Sim & Não & \\
\hline Diabetes mellitus $(n=1)$ & 1 & 0 & $<0,05$ \\
\hline Tabagismo $(n=16)$ & 2 & 14 & $>0,05$ \\
\hline \multicolumn{4}{|c|}{ Mecanismo da lesão $(n=127)$} \\
\hline Cortes $(n=37)$ & 6 & 31 & \multirow[t]{2}{*}{$>0,05$} \\
\hline Esmagamento $(n=90)$ & 18 & 72 & \\
\hline \multicolumn{4}{|c|}{ Classificação de Allen $(n=127)$} \\
\hline Tipo $1(n=41)$ & 6 & 35 & \multirow[t]{4}{*}{$<0,05$} \\
\hline Tipo $2(n=54)$ & 11 & 43 & \\
\hline Tipo $3(n=22)$ & 3 & 19 & \\
\hline Tipo $4(n=10)$ & 4 & 6 & \\
\hline
\end{tabular}

$56 \%$ mostraram preferir a desarticulação à amputação transóssea, e apenas 7\% preferiam ajustar o nível de amputação de acordo com o fechamento, na tentativa de preservar o máximo possível do comprimento do dedo. As características demográficas da amostra do presente estudo correspondem às das amostras de outros estudos, ${ }^{7,11,12}$ com predominância de homens jovens, com lesões por esmagamento, em que os dedos mais comumente afetados foram o segundo e o terceiro, conforme relatado por Torres-Fuentes et al. ${ }^{12} \mathrm{~A}$ diversidade de estudos com diferentes propostas de tratamento para o manejo dessas lesões demonstra a variedade de opiniões dos cirurgiões de mão, e dos cirurgiões plásticos e ortopédicos. Da mesma forma, a diversidade de classificações descritas nos últimos anos apenas complica a unificação dos critérios. ${ }^{13,14}$ Segundo a classificação de Allen, a maioria das lesões era do tipo 2, grupo em que tradicionalmente é considerado adequado o tratamento com retalhos de avanço, particularmente retalhos tipo V-Y de Atasoy, mas a irregularidade das bordas e as lesões comumente associadas limitam sua utilidade. As lesões de tipo 1 de Allen são tradicionalmente consideradas candidatas irrefutáveis para o tratamento conservador com a cicatrização seciundária, mas não os tipos 3 e 4 . No presente estudo, 25,3\% das amputações das pontas dos dedos tratadas por cicatrização secundária foram classificadas como 3 e 4 . Se considerarmos o total de amputações tratadas de forma conservadora, apenas 6,3\% $(n=5)$ necessitaram de intervenções cirúrgicas adicionais em virtude de complicações. Portanto, podemos afirmar que as lesões de maior extensão, às quais esse tratamento foi limitado, podem ser suscetíveis a essa modalidade técnica com preservação do comprimento. Os benefícios dignos de nota observados incluem: simplicidade, baixo custo, reprodutibilidade, disponibilidade de recursos materiais, fixação adequada, podendo a técnica ser usada com segurança em pacientes diabéticos e com história de tabagismo. No caso de pacientes pediátricos ou inquietos, não requer procedimentos dolorosos, nem a necessidade de 
realização em centro cirúrgico, evitando assim que o paciente seja submetido a riscos anestésicos. Por outro lado, a principal desvantagem é a longa duração do tratamento, que se traduz em um período relativamente longo de incapacidade para o trabalho, semelhante aos períodos relatados por van den Berg et al. ${ }^{7}$ Alguns pacientes ficaram inicialmente ansiosos com o odor e o exsudato produzidos nas primeiras semanas, mas ficaram satisfeitos após uma explicação. Fatores psicológicos já foram relacionados a impactos negativos na percepção funcional após o tratamento, maior intensidade da dor, e aumento dos dias de incapacidade para o trabalho. Desse modo, a identificação dos sintomas depressivos e o seu tratamento contribuirão para facilitar a recuperação. $^{15}$

O protocolo utilizado para cicatrização secundária demonstrou eficácia e segurança na população estudada, e permitiu que se demonstrasse a capacidade de regeneração tecidual, pelo menos nesta porção do corpo humano, questão polêmica e contemporânea. ${ }^{16}$

É importante salientar que a decisão de usar o gel de ketanserina é baseada na ampla disponibilidade comercial em nosso país e na experiência obtida no tratamento de úlceras vasculares e diabéticas. Entretanto, uma recente metanálise da Cochrane $^{17}$ apresentou resultados inconclusivos sobre sua eficácia em comparação com outras substâncias. Uma vez que outros agentes tópicos, como a vaselina e a sulfadiazina de prata, também foram descritos para o tratamento conservador da amputação da ponta do dedo, ${ }^{6}$ acreditamos que os resultados demonstrados no presente estudo não podem depender diretamente da ketanserina, mas de um microambiente rico em fator de crescimento adequado, controle de umidade e higiene, obtidos por meio de um acompanhamento atento e de intervenções precoces, quando necessário.

Com o objetivo de demonstrar a superioridade desse método, em relação à abordagem cirúrgica, são necessários estudos de desenho prospectivo e randomizado, a fim de avaliar objetivamente os resultados ao final de cada tratamento. Entretanto, em virtude da limitação de recursos humanos e materiais para a aplicação das técnicas de microcirurgia em todo o mundo, deve ser considerada como alternativa pelo cirurgião qualquer opção de tratamento que demonstre resultados estéticos aceitáveis, uma reintegração imediata à atividade laboral, e bons resultados funcionais.

\section{Conclusão}

A cicatrização secundária com o protocolo descrito demonstrou ser eficaz e segura no tratamento de amputações das pontas dos dedos classificadas como Allen tipos 1 e 2, e resultados satisfatórios foram obtidos em algumas lesões classificadas como Allen tipo 3; portanto, a cicatrização secundária deve ser considerada uma opção terapêutica até mesmo nas lesões de maior extensão.

\section{Suporte Financeiro}

Não houve suporte financeiro de fontes públicas, comerciais, ou sem fins lucrativos.

Conflito de Interesses

Os autores declaram não ter conflito de interesses.

\section{Referências}

1 Peterson SL, Peterson EL, Wheatley MJ. Management of fingertip amputations. J Hand Surg Am 2014;39(10):2093-2101

2 Wittstadt RA. Treatment options for distal tip amputation. In: Strickland JW, Graham TJ. Master techniques in orthopaedic surgery: the hand. 2nd ed. Philadelphia: Lippincott Williams \& Wilkins; 2005:323-328

3 Ipsen T, Frandsen PA, Barfred T. Conservative treatment of fingertip injuries. Injury 1987;18(03):203-205

4 Hoigné D, Hug U, Schürch M, Meoli M, von Wartburg U. Semiocclusive dressing for the treatment of fingertip amputations with exposed bone: quantity and quality of soft-tissue regeneration. J Hand Surg Eur Vol 2014;39(05):505-509

5 Mühldorfer-Fodor M, Hohendorff B, Vorderwinkler KP, van Schoonhoven J, Prommersberger KJ. [Treatment of fingertip defect injuries with a semiocclusive dressing according to Mennen and Wiese]. Oper Orthop Traumatol 2013;25(01):104-114

6 Krauss EM, Lalonde DH. Secondary healing of fingertip amputations: a review. Hand (N Y) 2014;9(03):282-288

7 van den Berg WB, Vergeer RA, van der Sluis CK, Ten Duis HJ, Werker PM. Comparison of three types of treatment modalities on the outcome of fingertip injuries. J Trauma Acute Care Surg 2012; 72(06):1681-1687

8 Allen MJ. Conservative management of finger tip injuries in adults. Hand 1980;12(03):257-265

9 Shauver MJ, Nishizuka T, Hirata H, Chung KC. Traumatic finger amputation treatment preference among hand surgeons in the US and Japan. Plast Reconstr Surg 2016;137(04):1193-1202

10 Li A, Meunier M, Rennekampff HO, Tenenhaus M. Surgical amputation of the digit: an investigation into the technical variations among hand surgeons. Eplasty 2013;13:e12

11 Sebastin SJ, Chung KC. A systematic review of the outcomes of replantation of distal digital amputation. Plast Reconstr Surg 2011;128(03):723-737

12 Torres-Fuentes CE, Hernandez-Beltran JA, Castañeda-Hernandez DA. Manejo inicial de las lesiones de punta de dedo: guía de tratamiento basado en la experiencia en el Hospital San José (91 casos). Rev Fac Med (Caracas) 2014;62(03):355-362

13 Park HC, Bahar-Moni AS, Cho SH, Kim SS, Park HS, Ahn SC. Classification of distal fingertip amputation based on the arterial system for replantation. J Hand Microsurg 2013;5(01):4-8

14 Champagne L, Hustedt JW, Walker R, Wiebelhaus J, Nystrom NA Digital Tip Amputations from the Perspective of the Nail. Adv Orthop 2016;2016:1967192

15 Bot AGJ, Bossen JKJ, Mudgal CS, Jupiter JB, Ring D. Determinants of disability after fingertip injuries. Psychosomatics 2014;55(04): 372-380

16 Miller TJ, Deptula PL, Buncke GM, Maan ZN. Digit Tip Injuries: Current Treatment and Future Regenerative Paradigms. Stem Cells Int 2019;2019:9619080

17 Broderick C, Pagnamenta F, Forster R. Dressings and topical agents for arterial leg ulcers. Cochrane Database Syst Rev 2020;1(01): CD001836 